\section{Evaluación multidimensional de los servicios de salud para adolescentes en centros de Atención Primaria en una comuna de Santiago}

\author{
CATALINA WILLIAMS DE T. a, FERNANDO POBLETE A. ${ }^{1}$, \\ FRANCISCA BALDRICH A. ${ }^{b}$
}

\section{Multidimensional assessment of public health care services for adolescents in Chile}

Background: Adolescents are an especially vulnerable age group in terms of behavioral issues and require skilled teams in health centers. Aim: To assess the quality of health services provided to teenage users in Primary Health Care. Material and Methods: A study of multiple cases was carried out in two family health centers in Puente Alto, Chile. Health services delivered to adolescents were evaluated from the provider's perspective, through qualitative design of focus groups and interviews to the care teams at each centre. For technical quality, comparing electronic records of two tracer conditions (prenatal care and depression) with technical standards established by Delphi methodology and from teenage users perspective, through a survey of service satisfaction. Results: In both centers, providers perceived a lack of training in adolescent care, a deficient preventive approach and a limited access to care. The technical evaluation showed an inappropriate recording of both tracer conditions. The instrument used to assess user satisfaction survey was reliable and showed that the best perceived issue was medical care and treatment, and the least perceived, was the access to the services. Conclusions: Professionals working in these health care facilities, feel unprepared to provide comprehensive approach to adolescents. The surveyed teenagers complained of limited access to care. Therefore this age group continues to be as a non-priority group for health care.

(Rev Med Chile 2012; 140: 1145-1153).

Key words: Adolescent; Adolescent Health Services; Primary Health Care.

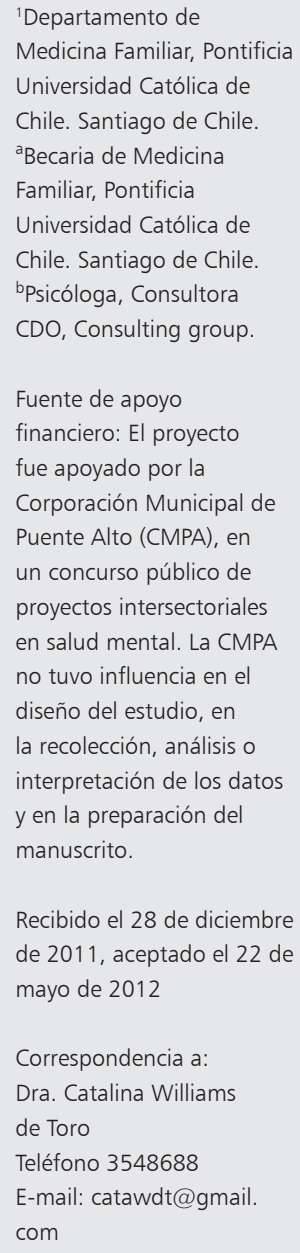

Recibido el 28 de diciembre de 2011, aceptado el 22 de mayo de 2012

L os adolescentes constituyen un grupo importante en la sociedad en número $y$, además, porque la evidencia sugiere que muchos problemas de la adultez comienzan en la adolescencia, estimándose que casi $2 / 3$ de las defunciones prematuras y $1 / 3$ de la carga total de morbilidad de los adultos pueden estar vinculadas a condiciones que se iniciaron en la juventud ${ }^{1}$. Un ejemplo constituye el inicio precoz del consumo de alcohol, que se asocia fuertemente a mayor probabilidad de dependencia en la edad adulta, sobrepasando el $50 \%$ si hay historia de alcoholismo en la familia, y el $30 \%$ si no hay historia familiar ${ }^{2}$.
En Chile, en el año 2004, la población adolescente representaba $25,7 \%$ de la población total (Robledo P. Que hay de nuevo en el Programa de Salud del Adolescente. III Congreso Chileno de Adolescencia). Algunos riesgos importantes son, por ejemplo, el consumo de último mes de tabaco en 35\%, alcohol en 37\% de los escolares entre 14 y 18 años, marihuana en 15\%, cocaína y pasta base en $3 \%{ }^{3}$. Por otro lado, el embarazo adolescente alcanza $16,3 \%$ del total de nacidos vivos el año $2009^{4}$, y la presencia de síntomas depresivos se observa con mayor frecuencia que la población adulta ${ }^{5}$ con ideación suicida en $20 \%$ de ellos en el año $2004^{6}$. 
Hay pocos estudios sobre servicios de salud a adolescentes, en un grupo en el que se sabe que su tasa de consulta es baja. Algunos estudios lo sitúan en no más de $50 \%{ }^{7}$ anual en Estados Unidos de Norteamérica, y en Latinoamérica sólo en $11 \%{ }^{8}$, con una tendencia importante en inequidad en acceso a salud según nivel socioeconómico en las Américas?.

La OMS promueve los servicios de salud amigables para adolescentes, una política integral de salud para los jóvenes, y su empoderamiento para que ejerzan su derecho a estos servicios ${ }^{10}$. Para lograrlo plantea ampliar la formación de los profesionales que trabajan con jóvenes, de cara a un enfoque preventivo ${ }^{11}$. En esa línea, en Chile el año 1999 se publica el primer documento sobre Políticas Públicas en Salud de Adolescentes y Jóvenes, y el año 2007 (Plan de Salud 2007. Corporación Municipal de Puente Alto. Santiago de Chile. Agosto 2006) se renueva el Programa adolescente con espacios de salud amigable, centrado en promoción y prevención en la Atención Primaria de Salud como un lugar estratégico para el acceso de este grupo, donde se ofrece servicios a nivel nacional, en el sector público, para cerca de $70 \%$ aproximado de la población del país a través de centros de salud familiar bajo la administración municipal.

El objetivo del presente estudio es evaluar la calidad de los servicios de salud prestados a usuarios de este grupo etario en Atención Primaria de Salud, conocer sus percepciones frente a estos servicios, así como las barreras y facilitadores que el Equipo de salud encuentra en la atención de adolescentes en una zona urbana de Santiago, Chile.

\section{Métodos}

Estudio de casos múltiples, conducido en dos Centros de Salud Familiar (CESFAM) de Puente Alto, Chile, que entre otros servicios entregan atención de salud a una población aproximada de 11 mil adolescentes en cada uno.

Se evaluó calidad de los servicios de salud usando métodos mixtos, integrando la perspectiva de la calidad técnica con metodología de condiciones trazadoras ${ }^{12}$, la perspectiva del usuario adolescente mediante encuesta autoaplicada, y la perspectiva de los prestadores a través de entrevista a informantes claves.

\section{Etapas del estudio}

1. Evaluación de calidad técnica: se midió con metodología de condiciones trazadoras ${ }^{13}$, a través de auditoria de ficha clínica electrónica (FCE) para depresión y control prenatal, seleccionadas por representar parte importante de los motivos de atención en el Centro de Salud de este grupo etario. Se definió un estándar técnico por metodología Delphi ${ }^{14}$, consultando a expertos de atención primaria y secundaria en base a protocolos de atención nacionales.

Se evaluó la FCE de todos los adolescentes atendidos por estas 2 condiciones durante 18 meses (enero de 2007 hasta junio de 2008). Se definió un grupo de parámetros que deben registrarse en la ficha clínica, sobre los cuales se evaluó la presencia o ausencia de su registro en la ficha posterior a la atención del paciente. Frente a la ausencia del registro, se asumió que este parámetro no fue evaluado en la consulta, reduciendo, por lo tanto, la calidad de ésta. Se definió un total de 36 criterios en depresión y 26 criterios en control prenatal (Tabla 1). Fue considerado bueno el registro igual o sobre el $80 \%$ de los parámetros y excelente el $100 \%$.

En la validación de datos, se decidió excluir de la base la variable "solicitud de TSH", pues su registro estaba en su mayoría ausente por factores administrativos externos al control de los profesionales.

2. Percepción de prestadores: se obtuvo una muestra intencionada de los equipos asistenciales de cada centro, tratando de representar todos los estamentos para realizar grupos focales. Adicionalmente, se entrevistó a los jefes de equipo y director de cada centro. Los grupos focales y entrevistas personales incluyeron los 4 aspectos de calidad según Donabedian ${ }^{15}$ (infraestructura, calidad técnica, relación interpersonal, acceso), fueron grabadas, transcritas y analizadas por un profesional entrenado y externo a los servicios evaluados. Se realizó análisis de contenido, extrayendo los puntos centrales de cada entrevista.

3. Evaluación de satisfacción desde la perspectiva del usuario: se aplicó la encuesta "Evaluación de satisfacción con la atención de salud" (encuesta aplicada en Chile, no publicada, Comunicación personal con los autores), auto-administrada. Contiene 49 preguntas sobre los 4 factores en calidad según Donabedian ${ }^{27}$. Se categorizó las preguntas según escala de Likert con 4 categorías 
Evaluación multidimensional de servicios de salud - C. Williams et al

Tabla 1. Criterios de calidad de registro en condiciones trazadoras

\begin{tabular}{|c|c|c|}
\hline & Depresión & Control prenatal \\
\hline 1 & Tristeza & Embarazo deseado \\
\hline 2 & Anhedonia & Educación de embarazada \\
\hline 3 & Irritabilidad & Educación, trabajo pareja \\
\hline 4 & Anergia & Edad pareja \\
\hline 5 & Cambio apetito & Reacción colegio \\
\hline 6 & Alteración sueño & Consumo de alcohol-drogas \\
\hline 7 & Cambio peso $>4 \mathrm{~kg}$ & Tabaco \\
\hline 8 & Alteración memoria y concentración & Deserción escolar \\
\hline 9 & Sentimiento de culpa & Violencia intrafamiliar \\
\hline 10 & Ideación suicida & Víctima de abuso sexual \\
\hline 11 & Intento suicida & Trastorno psicológico \\
\hline 12 & Síntomas psicóticos/maniacos & Embarazo adolescente de la madre \\
\hline 13 & Disminución rendimiento escolar & Muerte fetal o neonatal \\
\hline 14 & Autoagresiones & Abortos provocados \\
\hline 15 & Psicopatología familia & Parto prematuro \\
\hline 16 & Muerte familiar 1er grado & PEG o GEG (pequeño o grande para edad gestacional) \\
\hline 17 & Abuso alcohol/consumo drogas & Embarazo previo \\
\hline 18 & Deserción escolar & Edad embarazos previos \\
\hline 19 & Suicidio familia & Método anticonceptivo \\
\hline 20 & Depresión previa & Hemoglobina y hematocrito \\
\hline 21 & Conflicto escolar severo & Urocultivo y orina completa \\
\hline 22 & Muerte familiar últimos 6 meses & Consejería VIH \\
\hline 23 & Violencia intrafamiliar & Ecografía obstétrica 11-14 Hbd \\
\hline 24 & Abuso sexual & Ecografía obstétrica 21-24 Hbd \\
\hline 25 & Maltrato & Chile Crece Contigo* \\
\hline 26 & Separación pareja último año & \\
\hline 27 & Pérdida de madre antes 11 años de edad & \\
\hline 28 & Bulling & \\
\hline 29 & TSH & \\
\hline 30 & Médico & \\
\hline 31 & Psicólogo & \\
\hline 32 & Seguimiento telefónico & \\
\hline 33 & Familiar cercano & \\
\hline 34 & Padres comprometidos en tratamiento & \\
\hline 35 & Educación del tratamiento & \\
\hline 36 & Educación de efectos adversos de fármacos & \\
\hline
\end{tabular}

*Programa de protección de la primera infancia. 


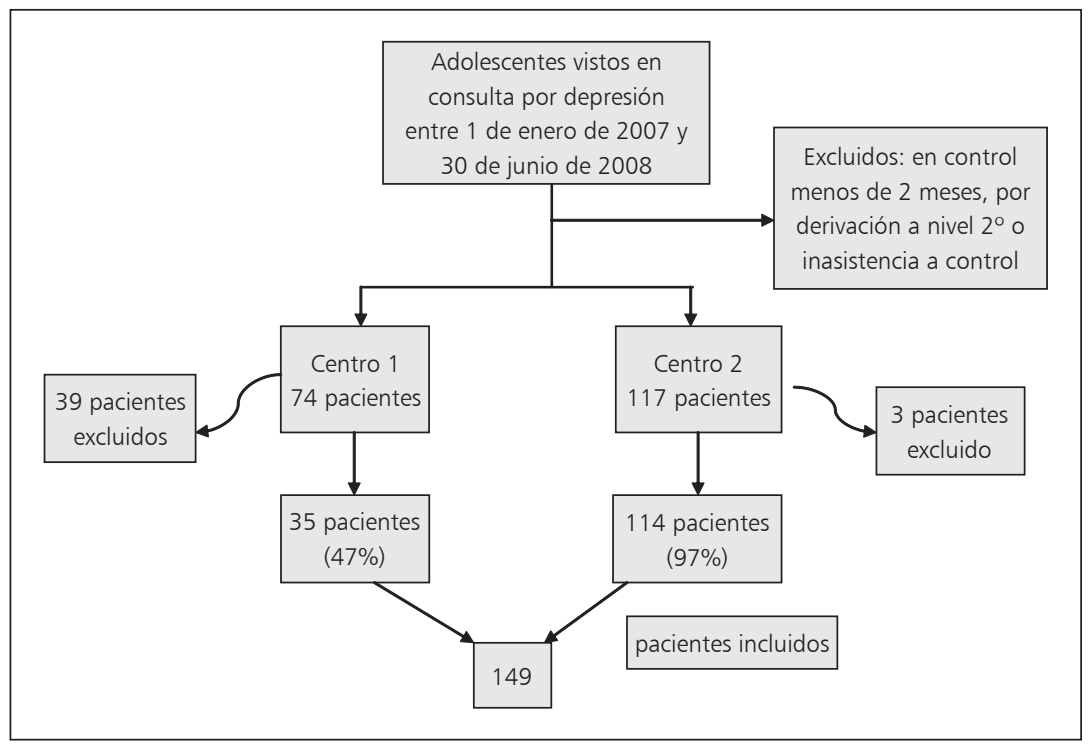

Figura 1. Algoritmo de fichas seleccionadas para evaluación técnica de depresión. por ítem, llevadas a puntajes estandarizados del 1 al 4 (muy de acuerdo-de acuerdo-en desacuerdomuy en desacuerdo). La muestra calculada con el programa SPSS en base a diseño de prevalencia desconocida, determinó 368 adolescentes entre 11 y 19 años a encuestar. Fue distribuida en la sala de espera de los sectores de los centros de salud participantes (Centro 1 y Centro 2) y en Unidad de Adolescencia (UDA) del Centro 1, en forma aleatoria durante 20 meses, en el período enero de 2008 hasta agosto de 2009. Los datos contenidos fueron examinados en el programa SPSS. Se realizó análisis de consistencia interna y por preguntas así como de la satisfacción global, colapsando categorías. No se realizó comparaciones entre centros, sino que un análisis descriptivo general para el total de la muestra.

Para evaluar la consistencia interna de la escala, se separó en 2 tipos de preguntas, expectativa (es decir, qué esperaría el adolescente respecto de la atención) y experiencia (es decir, lo que efectivamente evalúa como recibido en la atención). Para aquellas preguntas formuladas en sentido contrario (donde estar de acuerdo es negativo) la codificación para el análisis se realizó en forma inversa.

La encuesta a utilizar fue aprobada por el Comité de Ética de la Pontificia Universidad Católica de Chile en octubre del año 2008, y se permitió la entrega a menores de edad en las salas de espera.
Previo a la participación en las entrevistas y grupos focales se firmó un consentimiento informado.

\section{Resultados}

\section{Evaluación de calidad técnica}

Del total de pacientes incluidos en las fichas de depresión y control prenatal, se evaluó el registro de parámetros, y agrupó porcentajes de registro total en bueno $(>=80 \%)$, malo $(40 \%-79 \%)$, muy malo $(<40 \%)$.

Fichas de Depresión: se observó un porcentaje

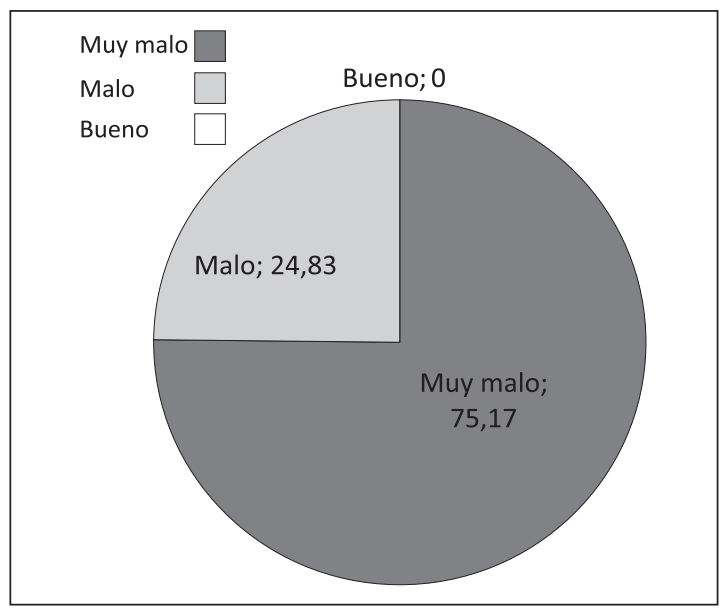

Figura 2. Registro en total de pacientes en control por depresión en porcentajes. 


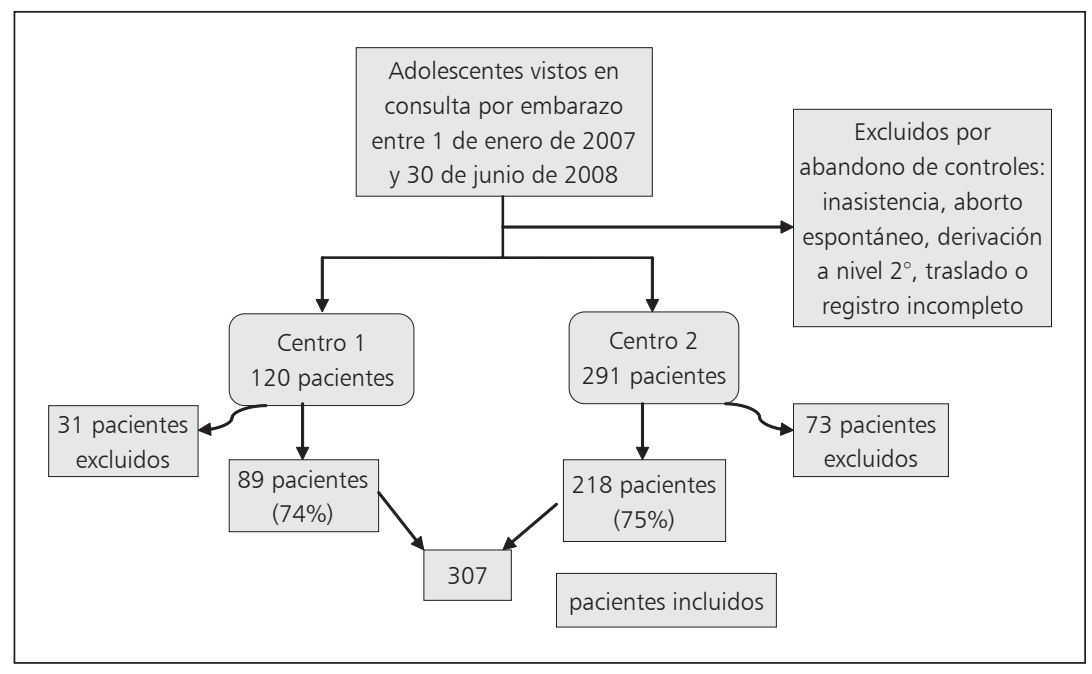

Figura 3. Algoritmo de fichas seleccionadas para evaluación técnica de control prenatal. de exclusión mayor de 50\% en Centro 1 comparado a Centro 2, donde fue 3\% (Figura 1). No hubo pacientes con registro de criterios bueno (Figura 2).

Fichas de control prenatal: se observó una pérdida similar en ambos centros (Figura 3), y algunos pacientes cumplieron con el estándar de buen registro, con un total de $9,8 \%$ en ambos centros (Figura 4).

\section{Percepción de prestadores}

En un centro se realizó 3 grupos focales y 5 entrevistas (incluida jefa de la Unidad de Adolescencia) y en el segundo centro, 4 grupos focales y 5 entrevistas.

Se destacó en ambos centros la ausencia de capacitación en políticas de salud amigables al adolescente, de enfoque preventivo y acceso limitado (Tabla 2).

\section{Evaluación de satisfacción adolescente}

Se entregó 450 encuestas, de las cuales se excluyó 64 , por estar incompletas (pérdida sobre $20 \%$ de las preguntas). El total de encuestas analizadas fue de 386.

Si se considera todas las preguntas de la encuesta, el alfa de Cronbach fue de 0,861. Para el sub-ítem de experiencia mejora la consistencia interna, con un valor de 0,878 . Para el sub-ítem de expectativa, el alfa de Cronbach fue de 0,336. La Tabla 3 muestra un resumen descriptivo de los resultados de la encuesta en experiencia y la Tabla 4 en expectativa.

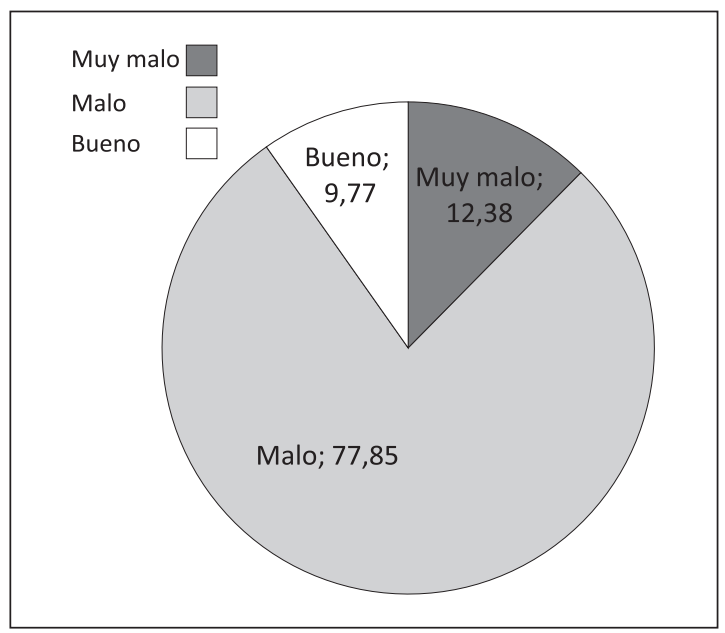

Figura 4. Registro en total de pacientes en control prenatal en porcentaje.

Los ámbitos mejor evaluados (aceptación mayor al 70\%) de las 4 categorías de calidad según la experiencia fueron:

1. Relaciones usuario-funcionario: destacado en la confidencialidad, amabilidad y educación.

2. Calidad técnica: sienten que reciben una atención de buena calidad profesional y administrativa, entienden las indicaciones y adhieren a tratamiento.

3. Infraestructura: el centro tiene el espacio adecuado, y está bien ubicado. 
Tabla 2. Resumen de resultados cualitativos según prestadores en ambos Centros de Salud

\begin{tabular}{|c|c|c|}
\hline & Centro 1 & Centro 2 \\
\hline Grupos focales & 3 & 4 \\
\hline Entrevistas & 5 & 5 \\
\hline $\begin{array}{l}\text { Parámetros evaluados } \\
\text { de calidad }\end{array}$ & \multicolumn{2}{|c|}{ Percepción de los prestadores en ambos Centros de Salud } \\
\hline Acceso & \multicolumn{2}{|c|}{$\begin{array}{l}\text { Adecuado en temas específicos por programas ministeriales de depresión y embarazo. Insuficiente } \\
\text { en el enfoque integral y patología general, ya que no son un grupo prioritario en la selección de } \\
\text { demanda diaria para horas médicas }\end{array}$} \\
\hline Infraestructura & \multicolumn{2}{|c|}{$\begin{array}{l}\text { Espacio limitado, no hay intención de cambios en la infraestructura. La UDA tiene su sala de espera, } \\
\text { pero está en un pasillo, por lo que no resguarda la confidencialidad de los adolescentes. Se realiza } \\
\text { actividades con adolescentes principalmente en ferias educativas escolares }\end{array}$} \\
\hline Calidad & \multicolumn{2}{|c|}{$\begin{array}{l}\text { Se sienten poco capacitados y no apoyados por la institución en esta necesidad, excepto matrona } \\
\text { y psicóloga de la UDA; sienten que la calidad es regular por esta razón, y ven que en los sectores, } \\
\text { los adolescentes son atendidos con enfoque curativo, y que el tiempo de la atención es insuficiente } \\
\text { para lograr una atención integral }\end{array}$} \\
\hline $\begin{array}{l}\text { Relaciones equipo } \\
\text { salud-adolescente }\end{array}$ & \multicolumn{2}{|c|}{$\begin{array}{l}\text { Buenas relaciones con adolescentes en la UDA, pero el equipo siente frustración por la sobrede- } \\
\text { manda y percepción de llegar a tan pocos adolescentes. Piensan que tiene buena recepción con } \\
\text { los adolescentes, porque no reciben reclamos de este grupo etario, y creen que tienen un rol de } \\
\text { referentes adultos para ellos. Otros ven una actitud desdeñosa, discriminadora desde los funcio- } \\
\text { narios hacia los adolescentes, y poca capacidad de mostrar empatía, establecerían mejor vínculo } \\
\text { los profesionales jóvenes }\end{array}$} \\
\hline
\end{tabular}

Tabla 3. Resumen descriptivo de los resultados de la encuesta de satisfacción a adolescentes en experiencia

\begin{tabular}{|c|c|c|}
\hline Encuesta & $\begin{array}{c}\text { De acuerdo } \\
(\%)\end{array}$ & $\begin{array}{c}\text { En desacuerdo } \\
(\%)\end{array}$ \\
\hline 1. Aquí me cuesta conseguir hora para el médico & 58,3 & 41,7 \\
\hline 2. Hay demora en los trámites que se hacen antes de recibir atención & 58,8 & 41,2 \\
\hline 3. Me atienden a la hora citada & 52,6 & 47,4 \\
\hline 4. Los remedios que me recetan me hacen sentir mejor & 82,3 & 17,7 \\
\hline 5. El personal del Centro de salud es amable conmigo & 90 & 10 \\
\hline 6. Es difícil conseguir hora para especialistas & 66,7 & 33,3 \\
\hline 7. El médico se da tiempo para explicarme bien las cosas & 79,1 & 20,9 \\
\hline 8. Los médicos toman en cuenta los sentimientos de la gente & 67,5 & 32,5 \\
\hline 9. El centro de salud tiene mal olor & 23,6 & 76,4 \\
\hline 11. Le entiendo al doctor cuando me explica lo que tengo & 82,6 & 17,4 \\
\hline 12. El Centro de salud se ve desordenado & 28,3 & 71,7 \\
\hline 14. Los doctores no explican por qué me piden exámenes & 38 & 62 \\
\hline 16. Yo volvería a atenderme aquí & 87,1 & 12,9 \\
\hline 17. Confío en que profesional no le va a contar a mis papás cosas mías & 76,2 & 23,8 \\
\hline 18. El profesional me inspira confianza para contarle mis problemas & 74,2 & 25,8 \\
\hline 19. El espacio en la sala de espera es suficiente & 65,3 & 34,7 \\
\hline
\end{tabular}


Evaluación multidimensional de servicios de salud - C. Williams et al

Tabla 3. (Continuación)

\begin{tabular}{|c|c|c|}
\hline 20. Cuando estoy angustiado el médico o la enfermera me escucha & 67,4 & 32,6 \\
\hline 21. Cuando me piden un examen me lo puedo tomar rápidamente & 45,6 & 54,4 \\
\hline 22. En el Centro de salud me resuelven los problemas por los que consulto & 73,1 & 26,9 \\
\hline 23. El lugar donde atienden los médicos es agradable & 90,6 & 9,4 \\
\hline 24. La ubicación del Centro de salud es buena para la gente & 87,2 & 12,8 \\
\hline 25. El Centro de salud se hizo chico para toda la gente que viene & 70 & 30 \\
\hline 26. A la gente que viene al Centro de salud la tratan mal & 25 & 75 \\
\hline 28. Me gusta la sala de espera & 66,8 & 33,2 \\
\hline 29. Es fácil perderse en este Centro de salud & 19,2 & 80,8 \\
\hline 30. No hay a quien preguntarle donde ir o que hacer & 27,7 & 72,3 \\
\hline 31. Los profesionales me atienden bien & 87,8 & 12,2 \\
\hline 32. Me gusta este Centro de salud & 85 & 15 \\
\hline 33. Los baños están limpios y en buenas condiciones & 60,1 & 39,9 \\
\hline 34. Yo cumplo las indicaciones que me da el médico & 89,4 & 10,6 \\
\hline 35. En el Centro de salud me atienden bien & 93,9 & 6,1 \\
\hline 36. El doctor me da una buena atención & 91,6 & 8,4 \\
\hline 38. Las preguntas que me hicieron cuando me atendían eran fáciles de entender & 87,2 & 12,8 \\
\hline 39. En la recepción me atienden bien & 82,8 & 17,2 \\
\hline 40. Muchas veces hay que comprar los remedios porque en el Centro de salud no hay & 63,6 & 36,4 \\
\hline 41. Tengo buena locomoción para llegar al Centro de salud & 79,6 & 20,4 \\
\hline 42. Si me falta un papel para atenderme en el Centro de salud me atienden igual & 40,8 & 59,2 \\
\hline 43. El Centro de salud está bien iluminado & 90 & 10 \\
\hline 44. En el Centro de salud hay un libro o persona que atiende los reclamos & 59,1 & 40,9 \\
\hline 45. El profesional me inspira confianza para contarle mis problemas & 73,4 & 26,6 \\
\hline 48. Hay material educativo en la sala de espera que me sirve para informarme & 58,3 & 41,7 \\
\hline 49. Me dan lo que necesito para mejorarme & 87,5 & 12,5 \\
\hline
\end{tabular}

Tabla 4. Resumen descriptivo de los resultados de las preguntas de expectativa de la encuesta de satisfacción adolescente

\begin{tabular}{|c|c|c|}
\hline & $\begin{array}{l}\text { De acuerdo } \\
(\%)\end{array}$ & $\begin{array}{l}\text { En desacuerdo } \\
(\%)\end{array}$ \\
\hline 2. Me gusta que me atienda la misma persona & 86,6 & 13,4 \\
\hline 13. Me molesta que interrumpan al profesional cuando me está atendiendo & 81 & 19 \\
\hline 15. Me gustaría estar sin mis papás cuando me atiendan & 50 & 50 \\
\hline 27. Es bueno que haya una sala de espera exclusiva para la gente de mi edad & 79,5 & 20,5 \\
\hline 37. Vendría a un control médico aunque no estuviera enfermo & 58,2 & 41,8 \\
\hline $\begin{array}{l}\text { 46. Me gusta que me hagan una entrevista completa además del problema por el que } \\
\text { consulto }\end{array}$ & 78,7 & 21,3 \\
\hline $\begin{array}{l}\text { 47. Es bueno que durante la consulta me entreguen información sobre el desarrollo } \\
\text { normal en la adolescencia }\end{array}$ & 93,7 & 6,3 \\
\hline
\end{tabular}


Tabla 5. Aspectos de experiencia evaluados en forma insatisfactoria

\begin{tabular}{|lcc|}
\hline & $\begin{array}{c}\text { De acuerdo } \\
\text { (\%) }\end{array}$ & $\begin{array}{c}\text { En desacuerdo } \\
\text { (\%) }\end{array}$ \\
\hline 1. Aquí me cuesta conseguir hora para el médico & 58,3 & 41,7 \\
\hline 3. Hay demora en los trámites que se hacen antes de recibir atención & 58,8 & 41,2 \\
\hline 4. Me atienden a la hora citada & 52,6 & 47,4 \\
\hline 33. Los baños están limpios y en buenas condiciones & 60,1 & 39,9 \\
\hline 44. En el Centro de salud hay un libro o persona que atiende los reclamos & 59,1 & 40,9 \\
\hline 48. Hay material educativo en la sala de espera que me sirve para informarme & 58,3 & 41,7 \\
\hline
\end{tabular}

Un aspecto mal evaluado (Tabla 5) fue el acceso tanto a nivel local (trámites, reclamos, medicamentos), como a nivel secundario (exámenes, control especialista).

\section{Discusión}

Uno de las primeras conclusiones que destaca este trabajo es el deficiente registro de los parámetros mínimos de la atención en las patologías estudiadas. Esto es preocupante porque reflejaría un estándar de calidad deficiente, ya que hay evidencia de que un buen registro se correlaciona directamente con la calidad del cuidado de la salud $^{16}$. Esto es consistente con la percepción de los prestadores, quienes reflejan una brecha en capacitación y dificultad para encuadrar la atención de acuerdo a las necesidades particulares de este grupo.

Llama la atención, también, la perspectiva de los prestadores sobre la necesidad de institucionalizar la importancia de la atención de adolescentes, lo que en último término se refleja en una relativa poca inversión de recursos en esta área, tanto en infraestructura, recursos humanos y capacitación.

Los adolescentes encuestados para evaluación de satisfacción usuaria revelan estar satisfechos en casi todos los aspectos y, mayoritariamente, contentos con los servicios recibidos. Contrariamente a lo que se esperaba y a lo expresado por los prestadores, los adolescentes perciben una buena atención en los aspectos más relacionales, refiriendo en algunas preguntas un nivel de satisfacción sobre el 90\%. En particular, es destacable el nivel de confianza en cuanto a confidencialidad y el alto nivel de comprensión de indicaciones entregadas por los profesionales. Es posible que un factor de confusión sea un nivel bajo de expectativas de los adolescentes, y por ello evalúen bien estos aspectos, o que efectivamente, más allá de lo percibido por los prestadores, la atención esté bien enfocada en los aspectos relacionales y comunicacionales. Esta es un área donde se requiere más estudio y quizás en el futuro incorporar metodología cualitativa con los adolescentes.

Además, destaca la evaluación positiva de la infraestructura, pese a la ausencia de espacios diferenciados para adolescentes en uno de los centros, y en el otro en forma parcial. De los aspectos peor evaluados, se encuentra principalmente el acceso a las prestaciones, y los tiempos de espera desde la recepción a la atención clínica.

Por otro lado, respecto de la encuesta, el énfasis en este análisis fue el de contrastar los resultados con las otras perspectivas utilizadas (del prestador y de la calidad técnica). De los datos de consistencia interna es evidente que el componente de expectativas tiene un mal desempeño, y pocas conclusiones se pueden obtener de esta perspectiva. Sin embargo, para el componente experiencia, la encuesta se desempeña muy bien y nos parece de utilidad para evaluar servicios de salud para adolescentes.

Este estudio presenta debilidades, entre las cuales el componente de evaluación de satisfacción del adolescente es una de las más importantes. Primero, pensamos que es necesario seguir mejorando el instrumento aplicado, aun cuando su consistencia interna es muy buena. Segundo, el estudio refleja las respuestas de aquellos que acuden al centro de salud y han tenido acceso a atención profesional, siendo este aspecto el más deficiente incluso para ellos. Tercero, al evaluar diferencias por centro 
de salud, no hubo diferencias importantes por preguntas, razón por la que se presentó un análisis global. En este punto pensamos que en futuros estudios es necesario realizar análisis comparativos con un mayor número muestral.

Este estudio es la primera aproximación diagnóstica de los servicios de salud para adolescentes con uso de metodología mixta, según nuestros registros, por lo que creemos es un aporte para la investigación en Chile en este tema. Si bien evalúa sólo dos centros de salud en la Región Metropolitana de Santiago, estos no se diferencian mucho, en términos de organización y estructura de recursos humanos, respecto de otros centros urbanos.

De este modo, la inversión en capacitación de recursos humanos, el énfasis en un adecuado registro y calidad de la atención, así como una mayor cobertura y accesibilidad parecen ser los aspectos más relevantes para la mejoría en la atención de los adolescentes en los centros estudiados, y probablemente en la Atención Primaria Chilena.

\section{Referencias}

1. Salud en las Américas, 2007. Volumen I-Regional.

2. Grant Bridget F. The Impact of a Family History of Alcoholism on the Relationship Between Age at Onset of Alcohol Use and DSM-IV Alcohol Dependence, Alcohol Health \& Research World 1998, 22: 144-8.

3. http://www.conace.cl/inicio/pdf/informe_ejecutivo_Estudio_Escolares_Conace2007_16-06-2008.pdf

4. Gobierno de Chile. Ministerio de salud. Situación actual del embarazo adolescente. http://www.minsal.gob.cl/ portal/url/item/aa54ffcc4262258de04001011e017558.pdf
5. Vicente B, Kohn R, Rioseco P, Saldivia S, Levav I, Torres S. Lifetime and 12-Month Prevalence of DSM-III-R Disorders in the Chile Psychiatric Prevalence Study. Am J Psychiatry 2006; 163: 1362-70.

6. EMSE 2004 (Encuesta Mundial de salud Escolar aplicada en Chile).

7. Marcell A, Matson P, Ellen J, Ford C. Changes In Adolescents' Use Of Routine Health Care Over Time. Journal of Adolescent Health 2009; 44 (2): S32-3.

8. Gobierno de Chile. Ministerio de salud. Política Nacional de Salud de Adolescentes y Jóvenes 2008-2015.

9. World Development Report, Making Services Work For Poor People. Washington, D.C. 2004.

10. World Health Organization. Global consultation on adolescent friendly health services. A Consensus statement. Geneva, 2002.

11. El Ambiente legislativo y de políticas para la salud de los adolescentes en América latina y El Caribe. OPS/Kellogg, Rodríguez y cols. 1998

12. Hermida J, Nicholas DD, Blumenfeld SN. Comparative validity of three methods for assessment of the quality of primary health care. Int J Qual Health Care 1999; 11 (5): 429-33.

13. National Academy of Sciences (U.S.), National Research Council (U.S.), National Academy of Engineering, Institute of Medicine (U.S.). Annual report, 1970-1971. Health Care and Services.

14. Jon Landeta, El método delphi: una técnica de previsión para la incertidumbre Barcelona: Ariel practicum, 1999.

15. Donabedian A. Quality assessment and assurance: unity of purpose, diversity of means. Inquiry 1988; 25: 173-92.

16. Osorio SG, Sayes VN, Fernández ML, Araya CE, Poblete MD. Auditoria médica: herramienta de gestión moderna subvalorada. Rev Med Chile [revista en la Internet] 2002; 130 (2): 226-9. 